\title{
Zur semantisch-pragmatischen Erweiterung der Abtönungs- funktion bei polnischen Modalpartikeln aus kontrastiver Sicht (Deutsch-Polnisch)
}

\author{
Magdalena Szulc-Brzozowska (Lublin)
}

\begin{abstract}
The present paper deals with the problem of the pragmatic equivalence between the German and the Polish modal particles. The author focuses on two pairs of particles, which have got an identical invariant meaning, such as the German particle aber and the Polish particle ale $(\dot{z})$ or the German particle $j a$ and the Polish particle no. The correspondence and the nocorrespondence of the use of this particles in the same sentence and situations context is considered with reference to their invariant meaning and to the position of the Polish particles in the sentence. The paper shows, that the pragmatic no-equivalence can result from the difference in the positions of the particles in German and Polish. The position of the Polish modal particles can be identical to the position of their homonyms in opposite to the German. The fact means that the position of the modal particles in Polish doesn't have a function of the categorization for speech parts, but it can influence on a semantic-pragmatic extension of the modal function of the particle.
\end{abstract}

\section{$1 \quad$ Fragestellung}

Der vorliegende Beitrag befasst sich mit der Problematik der Äquivalenz zwischen deutschen und polnischen Modalpartikeln/Abtönungspartikeln, genauer mit der Frage nach den Faktoren, die die pragmatische Äquivalenz bzw. Nicht-Äquivalenz zwischen miteinander korrespondierenden Partikeln des Deutschen und Polnischen herstellen. Als pragmatische Äquivalenz wird die kommunikative Gleichwertigkeit der Partikeln im gleichen Situationsund Satzkontext bezeichnet. Unter Korrespondenz der Partikeln, in dem vorliegenden Beitrag auch semantische Äquivalenz genannt, ist die Korrespondenz der übergreifenden Bedeutung der Partikeln gemeint. ${ }^{1}$ Die übergreifende Bedeutung bezieht sich auf alle Homonyme der jeweiligen Partikel, sowohl auf die Bedeutung der Partikel in ihrer primären syntaktischen Funktion, z. B. als Konjunktion, Adverb, Adjektiv oder Gradpartikel, als auch auf ihre Bedeutung in der Funktion als Modalpartikel (vgl. Szulc-Brzozowska im Druck).

Der Terminus modale Partikeln wird in der polnischen Fachliteratur in Bezug sowohl auf Partikeln, die den Abtönungspartikeln des Deutschen entsprechen, als auch auf Partikeln im weitesten Sinne gebraucht. Es handelt sich dann beispielsweise um den Ausdruck der Sprechereinstellung hinsichtlich des Wahrscheinlichkeitswertes des Aussageinhalts, also um Modalwörter (wie na pewno/gewiss, podobno/angeblich, możelvielleicht), um "fragende Partikeln"/sog. "partykuły pytajne" (z. B. czy ${ }^{2}$, kiedy/wann, kto/wer), die im Deutschen zum großen Teil den Fragepronomina entsprechen, auch um "Antwortpartikeln"/sog. "partykuły replikujące" (rzeczywiściel tatsächlich, oczywiście/selbstverständlich), die im Deutschen als Satzäquivalente funktionieren, oder um eine Art Modusoperatoren/sog. "partykuły wolitywne" (niech, niechby), die im Deutschen eigentlich keine partikelartige Äquivalenz haben. ${ }^{3}$ Es besteht kein einheitlicher Inhalt bezüglich des Begriffs modale Partikeln, weil die Elemente der Klasse der modalen Partikeln je nach Auffassung der Modalität bestimmt

\footnotetext{
${ }^{1}$ Zur übergreifenden Bedeutung der deutschen Modalpartikeln siehe z. B. Weydt/Hentschel (1983).

${ }^{2}$ Im Deutschen gibt es keine direkte Wiedergabe der polnischen "fragenden" Partikel $c z y$.

3 Nach Gruszczyński/Bralczyk (ed.)(2002: 183-184), nach Jodłowski (1971); Mirowicz (1947, 1948, 1949, 1956); Rieger (1957); Rachwałowa (1983); Szober (1923).
} 
werden. Es handelt sich um einen Oberbegriff, der ausschließlich semantisch determiniert ist. Dies hatte zur Folge, dass deutsche Modalpartikeln in älteren polnischen Grammatiken oder Handbüchern für Deutschlernende lange Zeit verschwiegen, d.h. meistens nicht übersetzt oder nicht ausreichend erklärt, sondern für eine ausschließlich deutsche Spezifik gehalten wurden. Man könnte sagen, dass die Existenz des analogen Phänomens im Polnischen dadurch quasi geleugnet wurde.

Das Polnische ist keine partikelarme Sprache; die Partikeln sind sowohl in der Literatur als auch in der Umgangssprache reichlich vorhanden. Erst im Rahmen einer kontrastiven Untersuchung der Modalpartikeln wurde festgestellt, dass im Polnischen die Abtönung nach deutscher Art auch in Form von Partikeln vorkommt. ${ }^{4}$ Die Vergleichsbasis bildete die pragmatische Funktion der deutschen Partikeln. Man hat im Polnischen Partikeln ausgesondert, die in pragmatischer Hinsicht den deutschen Modalpartikeln äquivalent sind. Das Kriterium der obligatorischen Stellung der Modalpartikel im Mittelfeld trifft auf "polnische Modalpartikeln" nicht zu. ${ }^{5}$ Zwischen manchen Partikeln des Deutschen und des Polnischen hat sich sogar eine Übereinstimmung unter dem formal-semantischen Aspekt ergeben. Der formal-semantische Aspekt betrifft die übergreifende Bedeutung der Partikeln und ihre primäre syntaktische Funktion.

Es lassen sich mehrere deutsch-polnische Paare von Partikeln finden, in denen die Partikeln eine gemeinsame syntaktische Funktion, meist als Adverb oder Gradpartikel oder auch Modalwort, haben, zugleich eine gemeinsame übergreifende Bedeutung tragen, dabei jedoch nicht in jedem Satztyp äquivalent sind. Zum Beispiel können ale und aber als adversative Konjunktion vorkommen; auch und też können als Adverb, Gradpartikel, Teil einer koordinierenden Konjunktion (sowohl ... als auch) funktionieren (die gemeinsame übergreifende Bedeutung heißt 'Konnex'); doch und jednak können als Konjunktion oder Adverb gelten (die gemeinsame übergreifende Bedeutung besteht in der Adversativität); eben und właśnie sind sowohl Gradpartikel als auch Temporaladverb (die gemeinsame übergreifende Bedeutung ist 'Unabänderlichkeit'/'Evidenz des Sachverhalts'); eigentlich und właściwie können als Adjektiv und Adverb fungieren (die gemeinsame übergreifende Bedeutung heißt 'im Grunde genommen, in Wirklichkeit'); einfach und po prostu (im Polnischen handelt es sich nicht um eine monolexikalische Partikel, sondern um eine Präpositionalgruppe) haben die primäre Funktion des Adjektivs bzw. Adverbs (die gemeinsame übergreifende Bedeutung ist 'Direktheit'); erst und dopiero sind auch Gradpartikel (die gemeinsame übergreifende Bedeutung besteht in einer Reihenfolgebestimmung); ja und no sind Antwortpartikeln (die gemeinsame übergreifende Bedeutung heißt 'Bejahung'); nur und tylko kommen auch als Adverb und Gradpartikel vor (die gemeinsame übergreifende Bedeutung heißt 'besonders wichtig'); ruhig und spokojnie gelten als Adverb (die gemeinsame übergreifende Bedeutung ist 'ungestört'); schon und już funktionieren als Temporaladverb und Gradpartikel (die gemeinsame übergreifende Bedeutung fokussiert die Gegenwärtigkeit des Sachverhalts); vielleicht und może können die Funktion des Modalwortes ausüben (die gemeinsame übergreifende Bedeutung heißt 'Möglichkeit'); wohl und chyba sind auch Modalwörter (die gemeinsame übergreifende Bedeutung lässt sich als 'Wahrscheinlichkeit'/'Vermutung' bezeichnen) ${ }^{6}$

Interessant ist bei diesen Paaren auch die Tatsache, dass im Polnischen, ähnlich wie im Deutschen, die Betonung der Partikel die Wortartzugehörigkeit ändern kann. Dies betrifft im Polnischen die Partikeln no, tylko, też, właściwie, właśnie und im Deutschen die Partikeln auch, denn, doch, eben, einfach, nur, vielleicht, wohl. Zum Beispiel:

Bring 'nur deine Katze in die Schule mit! (nur betont; Gradpartikel)

'Przynieś 'tylko swojego kota do szkoty!' (tylko betont; Gradpartikel)

vs.

\footnotetext{
${ }^{4}$ Es handelt sich um die Untersuchung von Szulc-Brzozowska (2002). Diese Arbeit enthält eine Beschreibung der deutsch-polnischen Äquivalenzbeziehungen zwischen den Modalpartikeln und die Charakteristik der "polnischen Modalpartikeln".

5 Zu den Kriterien der Abgrenzung der Modalpartikeln von anderen Partikeln im Deutschen vgl. z. B. Beerboom (1992); Hentschel/Weydt (1995); Stănescu (1989); Thurmair (1989); Weydt (1969); Weydt/Hentschel (1983). Das für die vorliegende Analyse gravierende Kriterium bildet die Nicht-Vorfeldfähigkeit der Modalpartikeln im Deutschen.

${ }^{6}$ Vgl. dazu Beispiele und die Beschreibung der Äquivalenz in Szulc-Brzozowska (2002).
} 
Bring nur deine 'Katze in die Schule mit! (nur unbetont; Modalpartikel) 'Przynieś tylko swojego 'kota do szkoły!'(tylko unbetont; Modalpartikel)

Zwischen den Partikeln (in den genannten deutsch-polnischen Paaren) lässt sich eine semantische Äquivalenz (bezüglich ihrer übergreifenden Bedeutung) und eine pragmatische Äquivalenz in manchen Satztypen sowie eine formale Übereinstimmung hinsichtlich der syntaktischen Funktionen der Partikeln feststellen.

Es entsteht die Frage, warum manche Partikeln nur in bestimmten Satz- bzw. Kontexttypen einander äquivalent sind, jedoch in anderen nicht, obwohl ihre übergreifende Bedeutung gleich ist.

Ziel des vorliegenden Beitrags ist es, den Abweichungen von der pragmatischen Äquivalenz zwischen den Partikeln nachzugehen, die eine gemeinsame übergreifende Bedeutung haben, also semantische Äquivalenz bezüglich der gleichen Wortartenzugehörigkeit aufweisen. Es wird gezeigt, dass die pragmatische Äquivalenz einerseits auf der semantischen Äquivalenz der Partikeln basiert und andererseits durch unterschiedliche Stellungsbedingungen der Partikeln in beiden Sprachen aufgehoben werden kann. Da die Stellung der Partikeln keinen Abgrenzungsfaktor bei der Bestimmung der Wortartenzugehörigkeit im Polnischen bildet, kommt es $\mathrm{zu}$ einer semantisch-pragmatischen Erweiterung der Abtönungsfunktion im Polnischen, die durch die Stellung der jeweiligen Partikel, somit durch die Emphase und die Thema/Rhema-Gliederung determiniert wird.

Den Hauptgegenstand meiner Analyse bilden zwei Partikelpaare ale( $\dot{z}) / a b e r$ und no/ja, die primär hinsichtlich ihrer gleichen übergreifenden Bedeutung und ihrer gleichen primären syntaktischen Funktion, erst dann wegen der Teil-Äquivalenz als Modalpartikeln zusammengestellt wurden. Es wird gezeigt, dass die pragmatische Funktion der polnischen Partikeln durch die Stellung ihrer Homonyme am Satzanfang so beeinflusst wird, dass die deutschen und polnischen Partikeln nur eine Teil-Äquivalenz aufweisen.

Ich stütze mich auf das Belegmaterial aus Szulc-Brzozowska (2002), das sowohl der Fachliteratur des Deutschen (z. B. Weydt et al. 1983; Weydt/Hentschel 1983; Thurmair 1989) als auch der Belletristik (deutsche und polnische) sowie der Befragung der jeweiligen Muttersprachler und der Aufnahme von Alltagsgesprächen entstammt.

\section{$2 \quad$ Zur Stellung der Partikeln im Polnischen ${ }^{7}$}

Die Stellung der Partikel im Polnischen ist - im Unterschied zum Deutschen - kein Kriterium zur Abgrenzung der Wortartenzugehörigkeit.

Das Polnische gehört zu den sog. nichtpositionellen Sprachen, d.h., die grammatische Funktion der Wortfolge ist sekundär. Die Wortfolge besitzt eine logische oder eine stilistische Funktion. Die logische Funktion beruht darauf, ein Satzelement durch die Verschiebung an den Satzanfang oder an das Satzende hervorzuheben (abhängig von Kontextbedingungen/Thematisierung/Rhematisierung). Man unterscheidet eine nicht-markierte/neutrale/primäre Stellung - Thema und danach Rhema (mit Satzakzent) - und eine markierte/sekundäre Stellung, die durch den Kontext bedingt ist - Rhema (mit Satzakzent) und danach Thema. ${ }^{8}$

Die Stellung der Partikeln mit der Abtönungsfunktion variiert im Polnischen je nach Partikel. Es gibt Partikeln, deren Stellung konstant ist, z. B. enklitische Partikeln, die sich an Fragewörter/Fragepartikeln anschließen (z. B. Gdzież jest moja ksiażka?/'Wo ist bloß mein Buch?'), oder Modusoperatoren, die je nach Partikel entweder am Satzanfang stehen oder dem Verb bzw. einer anderen Partikel hinzugefügt werden (z. B. Niech wyzdrowieje! oder Niechby

\footnotetext{
${ }^{7}$ In der deutschen Sprachwissenschaft ist die Diskussion über die Stellung der Modalpartikeln durch die Bestimmung ihrer Stellung vor dem Rhema oder an der Schnittstelle zwischen Thema und Rhema geprägt, z. B. nach Hentschel (1983, 1986) oder nach Thurmair (1989), nach Krivonosov (1965). In Ausnahmefällen sind die Modalpartikeln nach dem Rhema möglich, wenn das finite Verb das Rhema ausmacht oder das Rhema das Vorfeld bildet. Im Vorfeld können sie in Kombination mit dem Fragewort stehen. Jedoch wird generell die Ansicht vertreten, dass die Modalpartikel nicht erststellungsfähig ist, was gerade zum Abgrenzungskriterium der Modalpartikel von anderen Funktionen der Partikel wurde.

${ }^{8}$ Nach Gruszczyński/Bralczyk (eds.) (2002: 287-288).
} 
wyzdrowiat!/'Möge er gesund werden!' oder Mógłby wyzdrowiećl'Er könnte gesund werden'), sowie fragende Partikeln, die ausschließlich am Satzanfang stehen (z. B. Czy przyjdziesz do nas?/'Kommst du zu uns?'). Es gibt auch Partikeln, deren Stellung durch den emotionalen Wert der Äußerung bedingt ist (z. B. Ależ to jest piękny widok!/'Das ist aber eine schöne Aussicht!' oder Co ty tam wiesz?/'Was weißt du schon?', gemeint: nichts). Die Stellung hinter dem finiten Verb betrifft in intonatorischer Hinsicht eine neutrale Aussage. Die Verschiebung nach links, an den Satzanfang, also die initiale Stellung impliziert eine exklamative Intonation wie bei ale $\dot{z}$. Bei ale $(\dot{z})$ korrespondiert die Exklamation mit der Grundbedeutung der Abtönung, d. h. mit der Adversativität, dem Widerspruch, von daher nimmt ale $(\dot{z})$ ausschließlich die Position am Satzanfang ein im Gegensatz z. B. zu der Partikel tam. Die Partikel tam darf wegen der Bezeichnung der Beiläufigkeit, auch der Entfernung, nie an den Satzanfang gestellt werden.

Unter Partikeln, deren Bedeutung und Stellung einander bedingen, kann man solche unterscheiden, die ausschließlich am Satzanfang stehen, wie z. B. ale $(\dot{z})$, und solche, die ausschließlich die postinitiale Stellung einnehmen, wie z. B. tam.

Es gibt aber auch Partikeln, die sowohl die initiale als auch die postinitiale Stellung einnehmen können, wie z. B. przecież (On ma przecież rację oder Przecież on ma rację oder On ma racje przecież/'Er hat doch/ja recht'). Dies ist dann der Fall, wenn die Bedeutung der jeweiligen Partikel mit einem unterschiedlichen emotionalen Wert der Äußerung kompatibel ist. Die Verschiebung der Partikel nach rechts schwächt im Polnischen die emotionale Kraft der Aussage ab in Richtung 'Verunsicherung', 'Nachtrag'.

Die Stellung der Partikel korrespondiert somit auch mit der Bedeutung der Partikel im jeweiligen Satztyp. Zum Beispiel im Aufforderungssatz kann die Verschiebung der Partikel tylko nach rechts die Modifizierung der Illokution in Richtung 'Bitte' im entsprechenden Kontext (der Kontext ist entscheidend) bewirken im Gegensatz zur Nachdrücklichkeit, Warnung, Drohung im Falle der Verschiebung dieser Partikel nach links, z. B. Tylko zrób to!/'Mach es bloßß!' (sonst bekommst du eine Strafe) vs. Zrób to tylko!l'Mach es mal!' (um mehr wirst du nicht gebeten).

Inwieweit die Stellung der Partikel im Polnischen ihre semantisch-pragmatische Funktion modifiziert, wird im Folgenden an weiteren Beispielen dargestellt.

\section{Pragmatische vs. semantische Äquivalenz der Partikeln (Deutsch-Polnisch)}

\section{1 aber und ale $(\dot{z})$}

Die polnische Partikel ależ kann als eine stilistische Variante von ale gelten. Die Form ależ kann als eine Zusammensetzung aus der Partikel ale und der enklitischen Partikel -ize betrachtet werden, der ausschließlich eine verstärkende, emphatische Funktion zukommt $(-\dot{z} e$ funktioniert auch als subordinierende Konjunktion). Sie kann auch als stilistisch markiert gegenüber ale angesehen werden. Die stilistische Markierung geht in Richtung der veralteten Sprache und Literatursprache. $-\dot{Z} e$ als Komponente von ależ verhilft aber zur Abgrenzung der Funktion der Modalpartikel von der Funktion der Konjunktion, die ale auch ist. Ale und alez werden in dem vorliegenden Beitrag als eine Partikel betrachtet, wenn sie im jeweiligen Kontext einander abwechseln können, vom stilistischen Unterschied wird abgesehen.

Ale $(\dot{z})$ kommt, genauso wie aber im Exklamativsatz (in der Aussageform; ale $(\dot{z})$ auch im Exklamativsatz in der Frageform) vor. Die primäre syntaktische Funktion beider Partikeln aber und ale $(\dot{z})$ macht die adversative Konjunktion aus. Die Partikeln drücken einen Gegensatz zwischen zwei Propositionen aus, die sie verknüpfen, d. h. zwischen dem im Satz genannten Sachverhalt und dem vom Sprecher erwarteten. Zum Beispiel:

(1) Bern ist aber schön!l'Ale(ż) Berno jest piękne!'

oder

(1a) Ale(ż) jakie to Berno jest piękne!/'Wie schön ist doch Bern!'

vs.

(Warschau ist nicht schön.) Wie schön ist aber Bern! (aber gilt hier als Konjunktion) 
oder

(1b) $\quad$ Ale(ż) jak ty wygladasz!''Wie siehst du bloß aus!' (gemeint als Vorwurf, z. B. jemand sieht schmutzig oder schrecklich aus)

Die übergreifende Bedeutung der Partikeln, d. h. die Adversativität, bildet eine gemeinsame Grundbasis für die pragmatische Äquivalenz im Exklamativsatz (Aussageform). Die pragmatische Funktion von aber und ale $(\dot{z})$ kann jedoch je nach Kontext variieren. Im Deutschen handelt es sich um eine graduelle Bedeutung von aber, d.h., das Staunen des Sprechers bezieht sich auf den Grad der Eigenschaft, in dem Beispiel (1) der Schönheit der Stadt. Im Polnischen kann sich das Staunen auch auf den Sachverhalt selbst beziehen, ohne dass eine Eigenschaft graduiert wird. Zum Beispiel:

(2) Ale(z) to jest Bern!/'Das ist doch Bern!' (und nicht Madrid).

Der Abtönungsfunktion bei ale( $\dot{z})$ liegt der Wiederspruch auch im Imperativ- oder Fragesatz zugrunde. Dagegen tritt aber mit der Abtönungsfunktion in den Satztypen nicht auf.

W-Fragesatz:

(3) Czekam już 2 godziny. - Ale(ż) po co ty tu siedzisz? Idź do domu!

'Ich warte schon 2 Stunden. - Wozu/warum sitzt du auch hier? Geh nach Hause!'(gemeint: Du verlierst nur Zeit.)

(3a) Ależgdzie jest moja torba?

'Wo ist bloß/nur meine Tasche?' (Ich suche sie seit einer Stunde.)

vs.

Wo ist aber meine Tasche? (aber als Konjunktion) (Kontext: Deine Tasche liegt hier, aber wo ist meine Tasche?)

Im W-Fragesatz (3a), sowie im W-Ausruf (1b), sorgt ale( $\dot{z}$ ) für die Vordringlichkeit der Frage/des Ausrufs, die mit bloß/nur wiedergegeben werden kann, oder auch in (3) für ihre Rhetorizität, die im Deutschen mit auch ausgedrückt werden kann, wobei der Sachverhalt als unerfreulich markiert wird. Die W-Frage steht dem Ausruf nahe (vgl. 3a und 1b). Mit ale $(\dot{z})$ wird auf einen Widerspruch zwischen dem Sachverhalt und der vorherigen Annahme oder Aussage hingewiesen. Zum Beispiel in (3) wurde erwartet, dass der Hörer nicht länger wartet und in (3a), dass die Tasche gefunden wird. In (1a) wurde nicht erwartet, dass Bern so schön ist.

Im E-Fragesatz ${ }^{9}$ ist der Gebrauch von ależ ausgeschlossen. ${ }^{10}$ Es kann die Modalpartikel ale erscheinen. Im E-Fragesatz können je nach Kontext auch Zweifel, Unsicherheit, Distanz zum Ausdruck kommen. Mit ale und doch widerspricht der Sprecher der vorherigen Annahme oder Aussage.

E-Fragesatz:

(4) Eva: - Miałam dzisiaj mało czasu./'Ich hatte heute wenig Zeit.' (vorher wurde verabredet, dass Eva die Fahrkarte kauft)

Anna: - Ale czy kupiłaś bilet?/'(Aber) Du hast die Fahrkarte doch gekauft?' (der Fragesatz mit ale wird im Deutschen durch einen Aussagesatz mit fragender Intonation und der Partikel doch wiedergegeben)

vs.

Du hast aber die Fahrkarte gekauft? (aber gilt hier als Konjunktion)

Mit auch wird eine positive Antwort suggeriert, die die vorherige Annahme oder Aussage widerlegen soll.

(4a) Eva: - Powiedziałeś to już przecież.l'Du hast es doch schon gesagt.'

Anna: - Ale czy uważnie stuchasz?/'(Aber) hörst du auch aufmerksam zu?'

\footnotetext{
9 "E-Frage" bezeichnet eine Entscheidungsfrage, dagegen wird für eine Ergänzungsfrage die Abkürzung "WFrage" gebraucht.

${ }^{10}$ So ist das Ergebnis der Umfrage. Die Verteilung der Stimmen bei der Befragung ist die folgende: 36 Stimmen von insgesamt 45 halten eine E-Frage mit ależ für inkorrekt. Siehe dazu Szulc-Brzozowska (2002: 96).
} 
Im Imperativsatz wird mit ale $(\dot{z})$ ein Widerspruch ausgedrückt, der Ermunterung, Ermahnung oder Ungeduld implizieren kann. Im Deutschen ist eine Wiedergabe mit doch möglich.

Ale(z) siadaj prosze!!'Setz dich bitte doch hin!'

vs.

(Du wirst mir alles genau erzählen). Setz dich aber (zuerst) hin! (aber in der Funktion der Konjunktion)

Die Nicht-Äquivalenz von aber und ale $(\dot{z})$ mit der Abtönungsfunktion im Frage- und Imperativsatz, auch im Exklamativsatz in der Frageform oder auch in einem bestimmten Kontext im Exklamativsatz in der Aussageform, kann darin liegen, dass die Bedeutung von ale $(\dot{z})$ als Modalpartikel durch die Stellung am Satzanfang der Bedeutung von ale als Konjunktion nahe steht. Eine postinitiale Stellung der Modalpartikel ale im E-Fragesatz (also hinter dem finiten Verb) ist überhaupt nicht möglich. Diese Partikel steht im E-Fragesatz ausschließlich am Satzanfang, genauso wie die Konjunktion ale. Sie könnte eventuell für die Konjunktion gehalten werden. Für eine solche Klassifizierung würde die Tatsache sprechen, dass die Partikel ależ in diesem Satztyp nicht vorkommen kann. Die Annäherung der Abtönungsfunktion an die Bedeutung der Konjunktion bei ale $(\dot{z})$ erfolgt auch dadurch, dass die enklitische Partikel $-\dot{z} e$ bei ale wegen der stilistischen Markierung als Literatursprache, pathetische, veraltete Sprache im modernen Polnisch nicht nur im E-Fragesatz, sondern auch in anderen Satztypen ausgelassen wird. Die Abtönung von ale $(\dot{z})$ kann somit im Frage- und Aufforderungssatz das aber als Konjunktion zum Teil abdecken. Die semantischpragmatisch-syntaktische Nähe von ale $(\dot{z})$ als Modalpartikel und ale als Konjunktion kann auch dadurch bestätigt werden, dass die Konjunktion aber zur Wiedergabe der Aussagen mit ale neben einer Modalpartikel, z. B. doch, in diesen Satztypen verwendet werden kann und verwendet wird (diese Feststellung stützt sich auf die Analyse der Beispiele aus der Belletristik ${ }^{11}$ ). Es handelt sich bei der E-Frage um eine Art Nachfrage. In Sätzen mit aber als Konjunktion klingt der Widerspruch nachdrücklicher als in Sätzen mit aber als Modalpartikel. Die Nachdrücklichkeit kann auch bei ależ festgestellt werden und auf die initiale Position zurückgeführt werden. Zum Beispiel:

Ale przyjdzie Pan jeszcze?/'Aber Sie kommen doch wieder?'

Die Einschränkung der Abtönungsfunktion im Deutschen auf die Mittelfeldstellung und die Erweiterung der Abtönung auf die initiale Stellung im Polnischen kann ein Grund für die Nicht-Übereinstimmung von ale $(\dot{z})$ und aber sein. Man könnte behaupten, dass es im Polnischen im Unterschied zum Deutschen, z. B. im Falle von aber und ale $(\dot{z})$, zu einer semantisch-pragmatischen Erweiterung der Abtönungsfunktion dadurch kam, dass die Stellung der Modalpartikel und ihres Homonyms am Satzanfang durch die Emphase, Nachdrücklichkeit gekennzeichnet ist. Somit verwischen die Grenzen zwischen der Funktion der Modalpartikeln und ihres Homonyms.

Zusammenfassend lässt sich feststellen, dass die bindend-adversative Bedeutung der Konjunktion ale infolge der gleichen linearen Stellung wie die Modalpartikel ale $(\dot{z})$ ihre Semantik als Modalpartikel ziemlich stark beansprucht. Zusätzlich spielt die emphatische Funktion der Stellung am Satzanfang mit, so dass die pragmatische Nicht-Äquivalenz von aber und ale $(\dot{z})$ in manchen Satz- und manchen Kontexttypen das Ergebnis ist.

\section{2 ja und no $^{12}$}

No und ja können als eine Antwortpartikel fungieren. ${ }^{13}$ Ihre übergreifende Bedeutung bildet die Übereinstimmung des Sprechers mit dem Gesagten. No kommt als Modalpartikel in allen Satztypen vor, dagegen erscheint $j a$ als solche im Aussage- und Aufforderungssatz. Die formale und semantische Korrespondenz der Partikeln, d. h. die gemeinsame übergreifende Bedeutung und der gleiche Status als Bejahungswort garantieren keinesfalls die pragmatische Äquivalenz. Die pragmatische Äquivalenz zwischen ja und no betrifft den Aussagesatz in einem solchen Kontext, in dem einfach die Zustimmung des Sprechers ausgedrückt wird im

\footnotetext{
11 Nach Szulc-Brzozowska (2002).

12 Vgl. Szulc-Brzozowska, im Druck.

${ }^{13}$ No kommt als Antwortpartikel in der Umgangssprache vor.
} 
Unterschied zum Kontext, in dem sich der Sprecher lediglich auf ein gemeinsames Wissen von Sprecher und Hörer bezieht und den Sachverhalt als dem Hörer bekannt darstellt, z. B.

No on to zrobil'Er macht das ja' (Paraphrase: Ich gebe zu, er macht das.) vs.

Du kannst zu Hause üben. Du hast ja ein Klavier zu Hausel'Możesz ćwiczyć w domu. Masz przecież pianino $w$ domu' (Paraphrase: Ich erinnere dich an die Tatsache, dass du ein Klavier zu Hause hast.)

In (2) wird ja durch eine andere Partikel als no (nämlich przecież) wiedergegeben, weil nicht mehr nur die Zustimmung, sondern vor allem der Verweis auf den Sachverhalt, dass der Hörer ein Klavier zu Hause hat, thematisiert wird. Sowohl die Bedeutung der Bejahung als auch die Stellung der Partikel no am Satzanfang in (1) zeigen die semantische und syntaktische Nähe von no als Modalpartikel mit no als Antwortpartikel. Dies kommt auch deutlich in Aussagesätzen mit aufforderndem Charakter zum Ausdruck, in denen die pragmatische Funktion von no als nachdrückliche Bejahung durch die Stellung der Partikel erzeugt und somit durch die exklamative Interpretation erweitert wird, z. B.

$$
\text { No zobaczmy. (Pokaż!)/'(Nun,) Ich will das sehen. (Zeig es schon!) }
$$

Im Exklamativsatz besetzt no auch die Stelle am Satzanfang und drückt eine Zustimmung/Bejahung des Sachverhalts aus. Sowohl die Stellung von no als Modalpartikel, die der Stellung von no als Antwortpartikel gleicht, als auch die emphatische Intonation des Ausrufesatzes dienen der Verstärkung der Bedeutung 'Bejahung', wodurch die pragmatische Funktion von no als Modalpartikel im Exklamativsatz darin besteht, den Sachverhalt als einen solchen nachdrücklich zu bestätigen. Solche Exklamativsätze werden im Deutschen durch doch wiedergegeben. ${ }^{14}$ Zum Beispiel:

No jak ona pięknie śpiewa!/'Wie singt sie doch schön!' (vs. *Wie singt sie ja schön!)

vs.

Das ist ja grün! (Ich dachte, es wäre rot.)/'Ależ to jest zielone!'

vs.

No to jest zielone!/'Das ist erst grün!'

$J a$ kann im Exklamativsatz (Aussageform) vorkommen, wobei sich die Bejahung auch auf den gegebenen Sachverhalt bezieht, der aber nicht nur, wie bei no, als ein solcher bejaht wird, sondern als ein anderer als erwartet bezeichnet wird. In (5) handelt es sich also darum, dass der Sprecher sein Erstaunen über die Farbe 'grün' ausdrückt, wo er doch rot erwartet hat. Die Verwendung von no in (6) erzeugt die Bedeutung der Zustimmung zwischen dem Erwarteten und dem Sachverhalt, z. B. der Sprecher kann seine Verwunderung darüber ausdrücken, dass der Sachverhalt echt/schön grün ist, z. B. im Vergleich mit anderen Grünschattierungen. Die emphatische Intonation hebt den Sachverhalt als einen solchen hervor. No ist trotz der Ausrufeintonation nicht imstande, einen Wiederspruch zwischen dem Sachverhalt und dem Erwarteten auszudrücken. Dagegen kann durch ja eine solche Komponente eingeführt werden, weil ja nicht nur die Komponente der Bejahung enthält, sondern auch den Sachverhalt durch den Verweis des Hörers auf diesen Sachverhalt als unerwartet darstellt.

Auch im W-Fragesatz nimmt no die Erststelle ein. Seine pragmatische Funktion beruht auf der Erzeugung von Dringlichkeit, Ungeduld, Ermunterung, indem der erfragte Sachverhalt bejaht wird, z. B.

(7) No kiedy nadejdzie wiosna?/paraphrasierend: Sag mal/doch, wann der Frühling kommt bzw. Wann kommt bloß/ nur der Frühling?

vs.

* Wann kommt ja der Frühling?

In elliptischen W-Fragen kann no zur Rhetorizität führen, die eine positive Antwort suggeriert:

${ }^{14} \mathrm{Vgl}$. Szulc-Brzozowska (2002:132). 
Kto to jest? No kto? (dziekan naszego wydziału)

'Wer ist das denn?/Wer schon/ wohl? (der Dekan unserer Fakultät)'

Elliptische Fragen nehmen die Vorgängeräußerung auf, wobei no die bejahende Antwort impliziert. Die Rhetorizität ergibt sich zusätzlich aus der Expressivität der Bejahung.

$J a$ kommt im Fragesatz überhaupt nicht in Frage. Es würde den Sachverhalt als bekannt bezeichnen, was mit dem fragenden Satzmodus widersprüchlich wäre.

Im Imperativsatz kann die Stellung von no variieren. Zum Beispiel:

No czytaj! oder Czytaj no!/'Lies schon!' oder 'Lies doch!' oder 'Lies mal!' oder 'Lies nur!' (die Wiedergabe im Deutschen variiert je nach Kontext)

Es besteht keine Äquivalenz zwischen $j a$ und no in (9), weil no nicht vermag, solche Komponenten wie Aufmunterung, Dringlichkeit, Ungeduld oder sogar Abmilderung auszudrücken. Sie sind im Polnischen durch den Kontext und die Satzmelodie bedingt. No impliziert lediglich die Übereinstimmung des Sprechers mit der Proposition. Es kann sowohl die initiale Position (am Satzanfang) als auch die postinitiale, d. h. die hinter dem finiten Verb einnehmen. No kann im Aufforderungssatz wegen seiner möglichen Stellung im Mittelfeld, vor dem Rhema, problemlos als Modalpartikel angesehen werden, im Unterschied zum Aussagesatz, wo der Status von no als Modalpartikel wegen der gleichen Stellung von no als Antwort- und Modalpartikel bestritten werden kann.

Die pragmatische Äquivalenz zwischen ja und no kann im Aufforderungssatz nur unter der Voraussetzung bestehen, dass der Kontext eine Drohung oder Warnung impliziert, z. B.

(10) Sag 'ja nicht die Wahrheit!/'Nie powiedz no (tylko) prawdy!' (gemeint: Andernfalls wirst du die Konsequenzen tragen.)

(10a) Lies das Buch 'ja bis zu Ende!/'Przeczytaj no tę ksiażkę do końca!' (warnend gemeint: Andernfalls erfährst du die Pointe nicht.)

In (10) entsteht der warnende oder drohende Ton dadurch, dass der unerwünschte Sachverhalt negiert wird. Dies wird durch ja dem Hörer als bekannt unterstellt. Dagegen erscheint der Sachverhalt im nicht negierten Satz als erwünscht, wie in (10a). Der Hörer wird auf die Bekanntheit des Sachverhalts mit Nachdruck, durch die Betonung von ja im Imperativsatz, verwiesen.

Auch in (11) liegt es am Kontext, dass die Aufforderung als Warnung oder Drohung verstanden wird (im nicht negierten Imperativsatz), wo jedoch $j a$ kein Äquivalent für no bildet, z. B.

(11) Zepsuj no samochód!/'Mach 'bloß das Auto kaputt!' (Dann passiert was Schlimmes.)

In (11) wird der Sachverhalt als unerwünscht dargestellt, wobei dies nicht negiert wird. Der Verweis auf einen unerwünschten Sachverhalt (durch ja) ist daher nicht möglich.

Im E-Fragesatz kann no unterschiedliche Stellungen einnehmen. Je nach Kontext kann auch die pragmatische Funktion von no variieren. Es kann sich um eine Vergewisserungsfrage handeln, z. B.

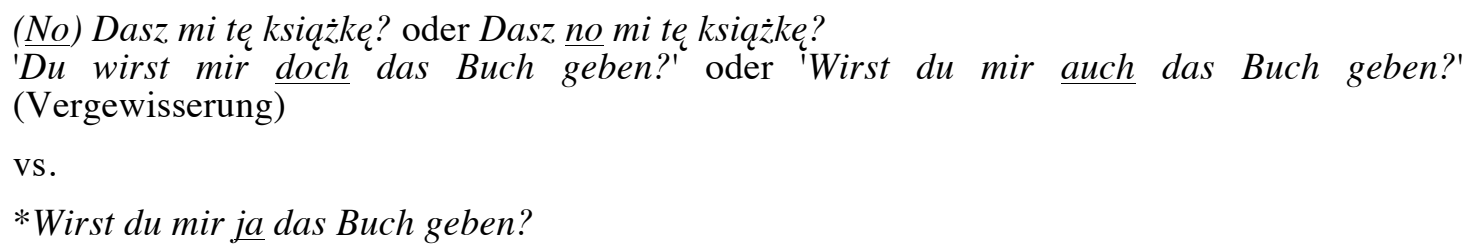

Im Deutschen können die Partikel auch und bei der Zweitstellung des Verbs die Partikel doch als Äquivalente erscheinen.

In der E-Frage, ähnlich wie im Aufforderungssatz, wird mit no Dringlichkeit erzeugt, und zwar die Dringlichkeit der Erwartung der Bejahung. Solche Fragen mit no am Satzanfang drücken oft Ungeduld aus. Dies zeugt von der semantisch-pragmatischen Nähe der Modalpartikel no zu no als Antwortpartikel. Die Komponente der Bejahung kann sich auch darin äußern, dass eine positive Antwort (eben die Bejahung des Hörers) vorausgesetzt wird, wodurch die Frage rhetorisch wird. 


\section{$4 \quad$ Zusammenfassung}

In dem vorliegenden Beitrag wurde die Aufmerksamkeit auf Gemeinsamkeiten der Partikelsysteme im Deutschen und Polnischen gerichtet, und zwar auf die gemeinsame primäre syntaktische Funktion der Partikeln, ihre gemeinsame übergreifende Bedeutung und sogar ähnliche Akzentverhältnisse bei manchen Modalpartikeln. Als unterschiedlich wurden Stellungsbedingungen dargestellt. Im Deutschen ist die obligatorische Stellung im Mittelfeld zugleich ein Abgrenzungsfaktor der Modalpartikel von ihrer anderen Funktion; im Polnischen gelten keine Beschränkungen auf das Mittelfeld, sogar können die Stellungsbedingungen jeder einzelnen polnischen Modalpartikel ihre pragmatische Funktion beeinflussen. Die Bedingtheit der Stellung der Partikel am Satzanfang im Polnischen, die mit der Emphase und Rhematisierung der Partikel zusammenhängt, wurde als ein Faktor gezeigt, der in einzelnen Kontext- oder Satztypen zur Nicht-Äquivalenz zwischen den Modalpartikeln (des Deutschen und Polnischen) mit der gemeinsamen übergreifenden Bedeutung beiträgt. Es handelt sich insbesondere um Partikeln, die in ihrer primären syntaktischen Funktion die initiale Stellung einnehmen, d. h. als Konjunktion oder Antwortpartikel. Es hat sich nämlich erwiesen, dass im Falle solcher Modalpartikeln, deren Homonyme die obligatorische Stellung am Satzanfang aufweisen und die die Satzanfangsstellung selbst einnehmen, die Bedeutung der Homonyme für die pragmatische Funktion der Modalpartikel prägend ist. Sogar kann diese Tatsache eine andere Positionierung der Partikel im Satz verhindern. Somit gilt die Bedeutung der Partikel mit Satzanfangspositionierung als Kernbedeutung.

Es wurde außerdem bemerkt, dass im Falle der Partikeln mit einer variablen Stellung ihre pragmatische Funktion auch variieren kann. Es handelt sich um die Thema/RhemaGliederung des Satzes im Polnischen, in der die initiale Position der Partikel durch Emphase, dagegen die Position im rechten Teil der Aussage durch Abmilderung gekennzeichnet ist. Somit konnte die These von einer semantisch-pragmatischen Erweiterung der Abtönungsfunktion durch die Stellungsbedingtheit anhand von Beispielen mit Partikeln unterschiedlicher Stellung (nicht nur mit Partikeln der initialen Stellung) belegt werden.

\section{Literatur}

Beerboom, Christiane (1992): Modalpartikeln als Übersetzungsproblem. Eine kontrastive Studie zum Sprachenpaar Deutsch-Spanisch. Frankfurt am Main: Lang. (= Heidelberger Beiträge zur Romanistik 26).

Gruszczyński, Włodzimierz/Bralczyk, Jerzy (eds.) (2002): Słownik gramatyki języka polskiego. Warszawa: WSiP.

Hentschel, Elke (1983): "Partikeln und die Wortstellung". In: Weydt, Harald (ed.): Partikeln und Interaktion. Tübingen, Niemeyer: 46-53. (= Germanistische Linguistik 44).

Hentschel, Elke (1986): Funktion und Geschichte deutscher Partikeln. Ja, doch, halt und eben. Tübingen: Niemeyer. (= Germanistische Linguistik 63).

Hentschel, Elke/Weydt, Harald (1995): "Die Wortarten des Deutschen". In: Àgel, Vilmos/Brdar-Szabó, Rita (eds.): Grammatik und deutsche Grammatiken. Budapester Grammatiktagung 1993. Tübingen, Niemeyer: 39-60.

Jodłowski, Stanisław (1971): Studia nad czesściami mowy. Warszawa: PWN.

Krivonosov, Aleksej (1965): "Die Wechselbeziehung zwischen den modalen Partikeln und der Satzintonation im Deutschen". In: Zeitschrift für Phonetik, Sprachwissenschaft und Kommunikation 18: 573-589.

Mirowicz, Andrzej (1947): "Przysłówki przy liczebnikach, zaimkach i rzeczownikach". In: Język Polski 27: 166-68.

Mirowicz, Andrzej (1948): "O partykułach, ich zakresie i funkcji". Biuletyn PTJ 8: 134-148.

Mirowicz, Andrzej (1949): "O swoistej funkcji pozagramatycznej partykuł". Język Polski 29/1: 30-34.

Mirowicz, Andrzej (1956): "Pojęcie modalności gramatycznej a kwestia partykuł". Biuletyn PTJ 15: 81-92.

Rachwałowa, Maria (1983): "Przysłówki metatekstowe i modalne w próbie języka naukowego humanistyki". In: Rocznik Naukowo-Dydaktyczny WSP w Krakowie 80, Prace Jezzkoznawcze IV: 133-142.

Rieger, Janusz (1957): "O partykułach". Język Polski 37/3: 179-188. 
Stănescu, Speranta (1989): "Zum Status der Partikeln im Deutschen und Rumänischen". In: Weydt, Harald (ed.): Sprechen mit Partikeln. Berlin/New York: de Gruyter. 267-275.

Szober, Stanisław (1923): Gramatyka jezzyka polskiego. Lwów: Książnica Polska.

Szulc-Brzozowska, Magdalena (2002): Deutsche und polnische Modalpartikeln und ihre Äquivalenzbeziehungen. Lublin: Towarzystwo Naukowe KUL.

Szulc-Brzozowska, Magdalena (im Druck): "Zur Problematik der semantischen und pragmatischen Äquivalenz von Partikeln am Beispiel des deutschen ja und des polnischen no". Erscheint in: Lubelskie Materiały Neofilologiczne 34: 85-96.

Thurmair, Maria (1989): Modalpartikeln und ihre Kombinationen. Tübingen: Niemeyer. (= Linguistische Arbeiten 223).

Weydt, Harald (1969): Abtönungspartikel. Die deutschen Modalwörter und ihre französischen Entsprechungen. Bad Homburg: Gehlen. (= Linguistica et Litteraria 4).

Weydt, Harald (ed.) (1983): Partikeln und Interaktion. Tübingen: Niemeyer. (= Germanistische Linguistik 44 ).

Weydt, Harald /Hentschel, Elke (1983): "Kleines Abtönungswörterbuch". In: Weydt, Harald (ed.): Partikeln und Interaktion. Tübingen, Niemeyer: 3-24.

Weydt, Harald et al. (1983): Kleine deutsche Partikellehre. München: Klett. 